\title{
Phase transitions in two-dimensional colloidal particle system observed in Langmuir trough
}

\author{
Bo-Jiun Lin, Li-Jen Chen* \\ Department of Chemical Engineering, National Taiwan University, Taipei 10617, Taiwan, ROC \\ Received 25 June 2005; received in revised form 24 October 2005; accepted 27 October 2005 \\ Available online 13 December 2005
}

\begin{abstract}
Langmuir trough is applied as a platform to study the two-dimensional colloidal system. Polystyrene (PS) particles are distributed at decane/water interface in Langmuir trough. Digital video microscopy method is used to evaluate pair distribution function $g(r)$ of the system by directly measuring particle locations in each frame of images. In addition, the phase of the system is determined by calculating its structure factor. A sequence of phase transitions of the system is observed by varying particle density at $20^{\circ} \mathrm{C}$.
\end{abstract}

(C) 2005 Elsevier B.V. All rights reserved.

Keywords: Phase transitions; Two-dimensional colloidal system; Polystyrene particle; Structure factor

\section{Introduction}

It has been a wide interest in the behavior of particle monolayer at a fluid/liquid interface for several decades [1-12]. In 1968, Sheppard and Tcheurekdjian had estimated the strength of steric barriers between stabilized spherical polymer particles at the air/water interface from the measurement of isotherms of surface pressure and surface area for particle monolayers [2]. Aveyard et al. had explored the capability of particles at interfaces to modify the stability of foams and emulsions $[10,11]$. The aggregation of particles at the air/water interface as well as aggregation morphology had also been reported [4].

A typical way to study two-dimensional colloidal systems is to confine colloidal particles between two glass slides. The gap of separation between two slides is about 1.2 times larger than the particle diameter. If the gap of separation is smaller than $\sim 1.2$ times particle diameter, the colloidal particles become immobile. Because of this limitation, particle still can move along the vertical direction slightly. Therefore, this type of systems is usually called "quasi-two-dimensional system." Phase transitions in this quasi-two-dimensional system had been observed with different kinds of colloidal particles, such as, silica and PMMA particles [6,7]. Since the separation is always slightly

\footnotetext{
* Corresponding author. Tel.: +886 2 23623296; fax: +886223623040.

E-mail address: ljchen@ntu.edu.tw (L.-J. Chen).
}

larger than the particle diameter, some three-dimensional effect may occur. Besides, the glass slides also cause some boundary effect, for example, wall drag effect [6]. The experimental setup of the system is quite complicated and hard to assemble [6,7].

The Langmuir trough is also a common platform for studying two-dimensional colloidal system. Colloidal particles usually stay at interface in a form of monolayer. Aveyard et al. [10,11] have reported some properties about particle monolayer which was made of $2.6 \mu \mathrm{m}$ charged polystyrene particles at oil/water interface and at air/water interface.

In this study, we apply Langmuir trough as an alternative to observe phase transitions of two-dimensional colloidal particles. Polystyrene particles of $2 \mu \mathrm{m}$ diameter are distributed at decane/water interface in the Langmuir trough. For polystyrene particles, a wide variability of particle sizes and functional group grafted on the particle surface are available commercially. Digital video microscopy method was used to evaluate pair distribution function $g(\vec{r})$ of the system by directly measuring particle locations in each frame of images. In addition, the phase of the system can be further determined by calculating its structure factor.

\section{Experimental details}

The experiment was performed at decane/water interface on a Langmuir trough (NIMA technology, 611D) equipped 


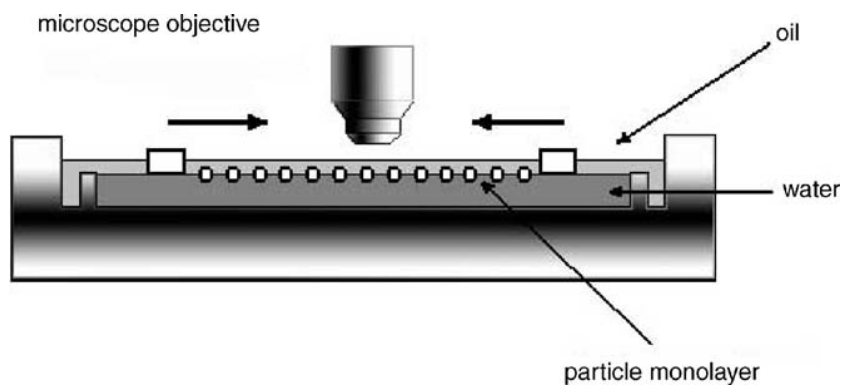

Fig. 1. Schematic representation of the experimental setup: the Langmuir trough and an upward microscope placed right above the trough for in situ observation.

with liquid-liquid option. Decane with 99\% purity was purchased from Merck and used as received. Water was purified by double-distillation and then followed by a PURELAB Maxima Series (ELGA, LabWater) purification system with the resistivity always better than $18.2 \mathrm{M} \Omega \mathrm{cm}$. The injected solution was prepared with polystyrene (PS) spheres grafted with sulfate group with diameter $2 \mu \mathrm{m}$ (Duke Scientific Company) mixed with isopropanol (IPA) as a spreading solvent at the volume ratio $1(\mathrm{PS}): 2(\mathrm{IPA})$ or $1(\mathrm{PS}): 3(\mathrm{IPA})$.

The experimental setup is shown in Fig. 1. An upward microscope (Olympus, BXFM) equipped with long-working distance objectives with different magnifications $(5 \times, 10 \times, 20 \times, 50 \times)$ was set up on the trough for in situ observation. The observation was performed in dark field method.

Several different volumes of solutions were injected into decane/water interface when the barriers were fully opened. Different reduced densities could be obtained by compressing the barriers. The reduced density $\rho^{*}$ was measured in units of particle number per frame area normalized by cross-section area of particle:

$\rho^{*}=N \frac{\pi \sigma^{2}}{4 A}$

where $N$ is number of particles in each frame, $\sigma$ is particle diameter and $A$ is the frame area. A long time observation was performed to examine the equilibration process of the system. After the particles solution was injected onto interface, we recorded more than 100 frames of images every $1 \mathrm{~h}$ with a time interval of $10 \mathrm{~s}$. There was no detectable change of structure factor after $1 \mathrm{~h}$. That implies the system would reach equilibrium in $1 \mathrm{~h}$. Therefore, all the data in this study were collected after the system was left to equilibrate for at least $2 \mathrm{~h}$ at $20^{\circ} \mathrm{C}$. The whole process was performed more than three times and the data were reproducible.

The images were captured by a monochrome charged coupled device (CCD) video camera (Sony Corporation) mounted on the microscope and grabbed by a frame grabber (NI-1409, National Instrument) and then recorded by a personal computer for data analysis.

A standard image process was applying to extract the information about positions of particles. We closely followed the method used by Crocker and Grier [9]. After the positions of particles were determined, the structure factor can be calculated from the following equation:

$S(\vec{k})=\rho \int \mathrm{e}^{-i \vec{k} \cdot \vec{r}} h(\vec{r}) \mathrm{d} \vec{r}$

which is the Fourier transform of $h(\vec{r})=g(\vec{r})-1$, where $\vec{r}$ is the position vector in the real space and $\vec{k}$ is the position vector in the reciprocal space. The radial distribution function $g(\vec{r})$ is

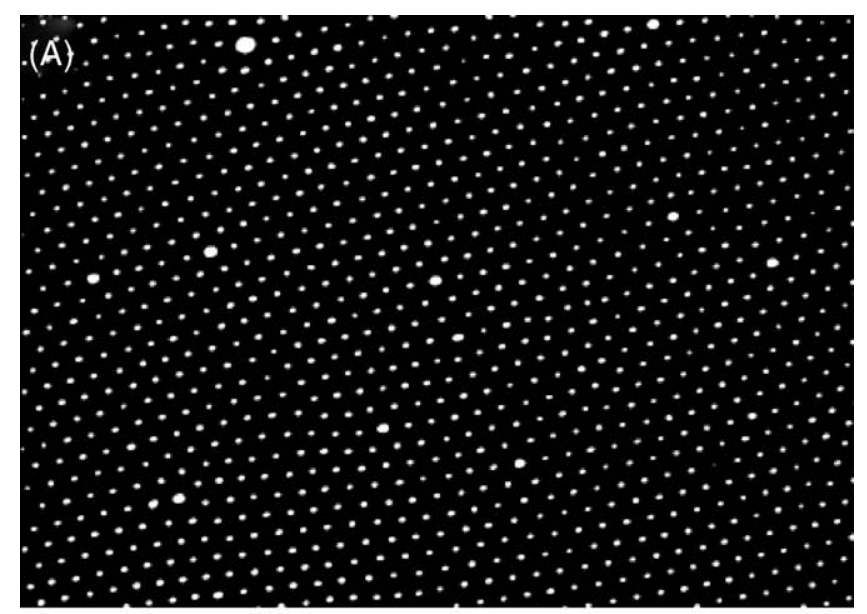

(B)
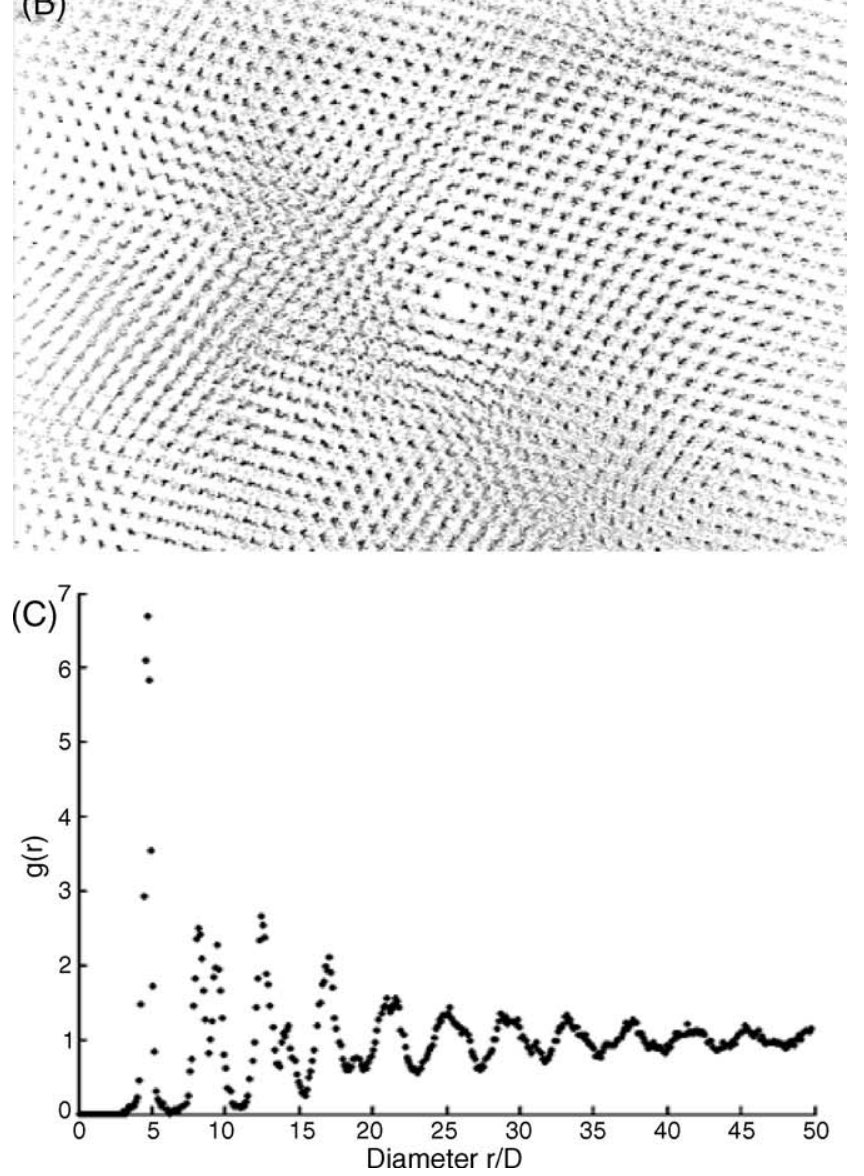

Fig. 2. (A) An image captured from CCD at reduced density 0.0432; (B) the radial distribution function determined from (A) in two dimensions, and (C) in one dimension. 
(A)

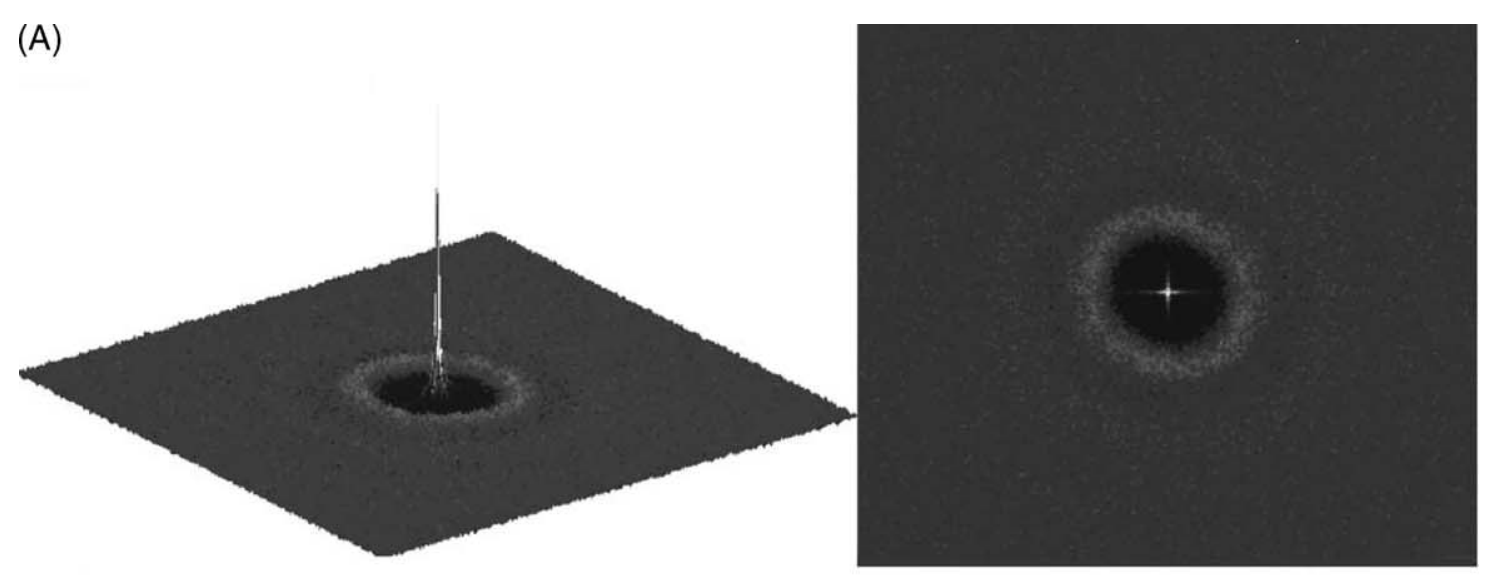

(B)
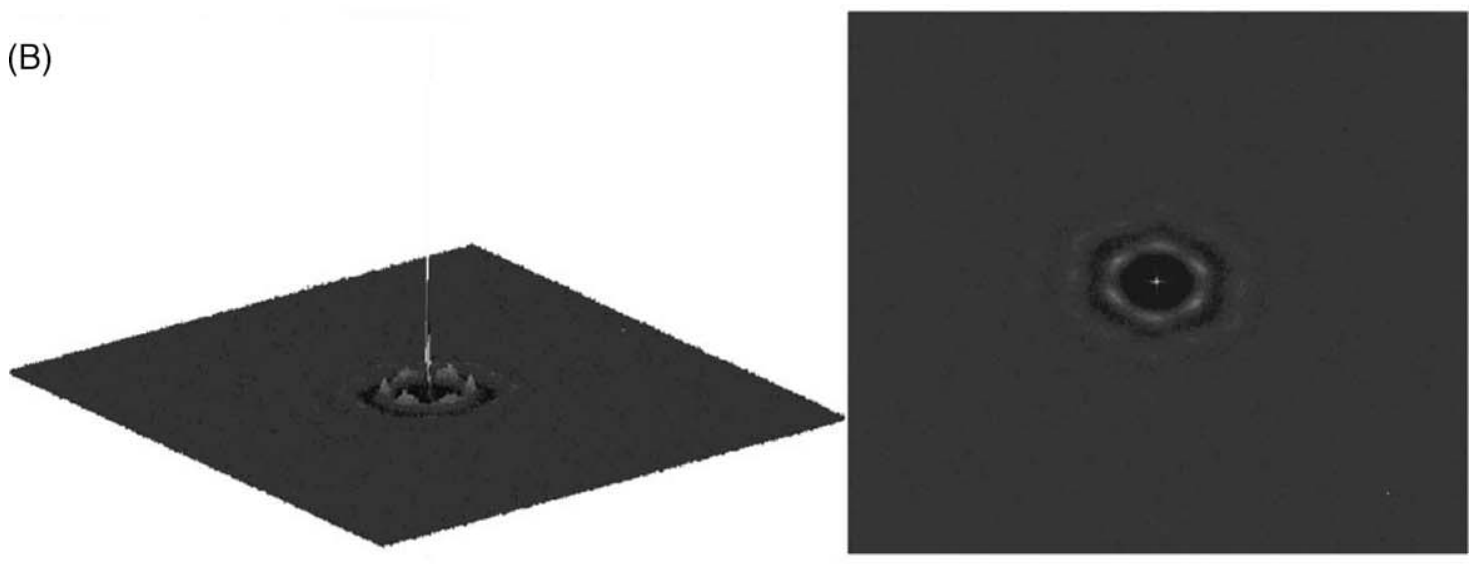

(C)

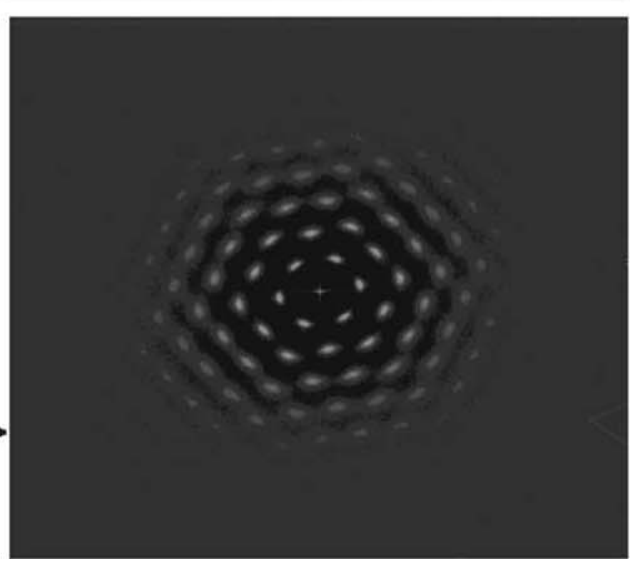

Fig. 3. Structure factor in three dimensions (left column) and in two dimensions (right column) of three different phases: (A) liquid phase, (B) hexatic phase and (C) solid phase.

calculated by:

$g(\vec{r})=\rho^{-2}\left\langle\sum_{i} \sum_{j \neq i} \delta\left(r_{i}\right) \delta\left(r_{j}-r\right)\right\rangle$

where $\delta(r)$ is the delta function.

\section{Results and discussion}

Fig. 2(A) illustrates an image captured from $\mathrm{CCD}$ at reduced density 0.0432. Fig. 2(B) and (C) demonstrate the radial distribution function, respectively, in two dimensions and in one dimension based on Eq. (3) according to the data extracted from Fig. 2(A). The radial distribution function is necessary for structure factor calculation in Eq. (2). Fig. 2(B) shows the calculated results for the radial distribution function in two dimensions. The distance of the first peak in the radial distribution function in one dimension stands for the nearest neighbor distance, which is about five times diameter shown in Fig. 2(C). Fig. 3 shows the typical results of the structure factor for three different phases: liquid, solid and hexatic phase, in two dimensions (right column) as well as in three dimensions (left column). The diffraction pattern for liquid phase is an isotropic ring, as shown in Fig. 3(A), a hexagonal ring for the hexatic phase, as shown 

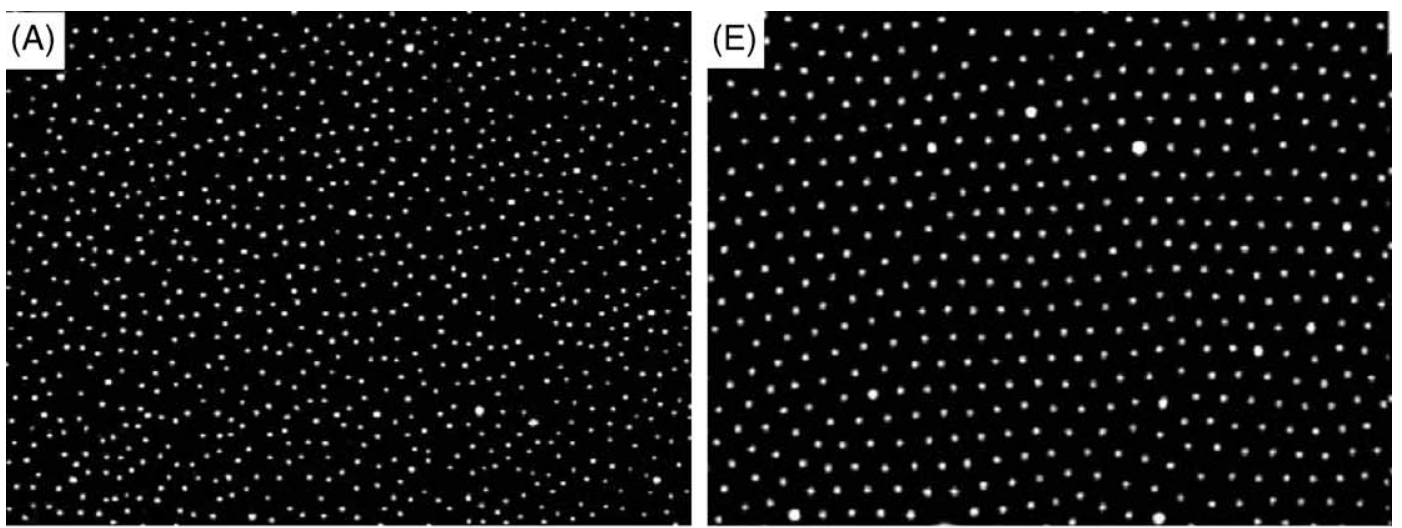

(B)
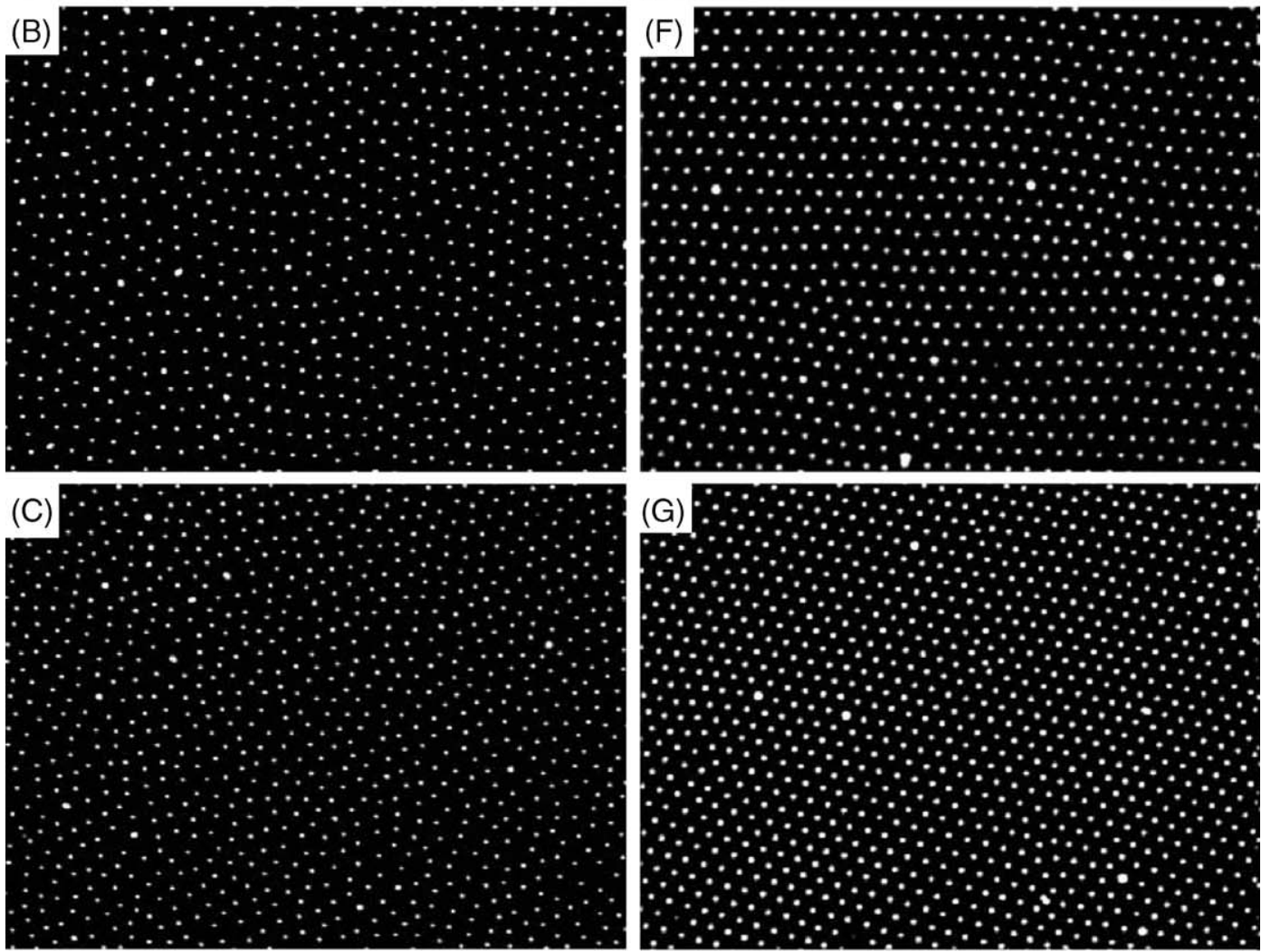

(D)

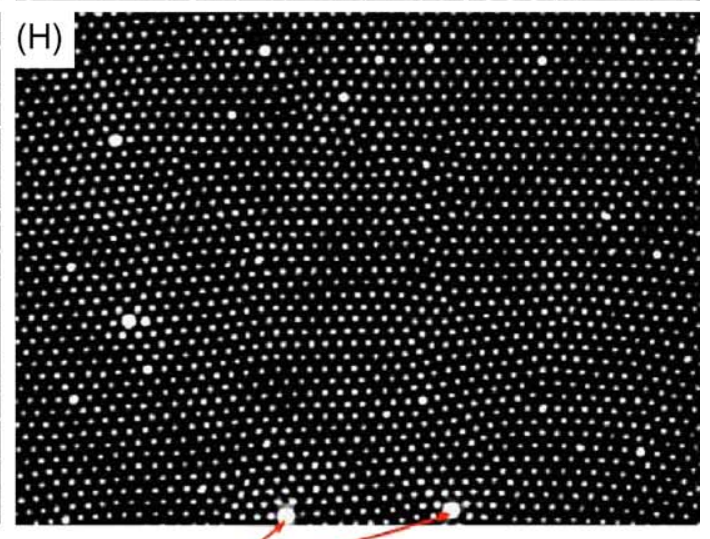

Aggregations of particles

Fig. 4. Sample images of the two-dimensional charged polystyrene particles at decane/water interface at eight different reduced densities: (A) 0.0044 , (B) 0.0073 , (C) 0.0121, (D) 0.0157, (E) 0.0223, (F) 0.0365, (G) 0.0432 and (H) 0.0856 . 

in Fig. 3(B) and six distinct spots for solid phase, as shown in Fig. 3(C). The phase of the system was determined simply by its diffraction pattern. At least 50 frames of images were calculated and averaged over at each density to enhance the quality of structure factor.

To start with the experiments, the air/water interface was chosen as the platform to study the particle monolayer. However, it is difficult to spread particles efficiently onto water surface. Aveyard et al. [10] pointed out that it is necessary to have a low concentration of electrolytes in the aqueous sub-phase for spreading particles at air/water interface. The addition of electrolytes to the system would also cause aggregation problems at high densities [10]. In contrast, colloidal particles can be easily spread onto oil/water interface without adding any electrolyte to aqueous sub-phase. In addition, colloidal particles are much more stable at oil/water interface than at air/water interface. Binks $[12,13]$ pointed out that the stability of suspending particles at the interface is strongly related to the contact angle between the particle and the interface. He found that the particle is most strongly sticking to the interface for the contact angle equal to $90^{\circ}$. Either larger or smaller than $90^{\circ}$, the desorption energy is rapidly decreased. Higher desorption energy makes particle more likely to stay at interface. In other words, the lower desorption energy means the particle is easier to detach from interface [13]. Note that the contact angle of polystyrene particle at air/water interface is usually less than $20^{\circ}$ and that at oil/water interface is larger than $70^{\circ}$ [10]. Therefore, the decane/water interface is used in this work to spread PS particles of $2 \mu \mathrm{m}$ in diameter.

Scattering pattern can be obtained by calculating structure factor that could be applied to distinguish phase transitions within the density range of the system. Fig. 4 shows the images at eight different reduced densities ranging from 0.0044 to 0.0856 and the corresponding structure factors are shown in Fig. 5. It is quite difficult to avoid the aggregation of particles that had been often found in literatures, for example, Fig. 7 in reference [10]. In fact, the aggregation problem would only occur in the system of high densities. Furthermore, the number of the aggregation sites, as shown in Fig. 4(H), is so small that has no influence on determining the phase of the system.

At low densities, say $\rho^{*}=0.0044$, the structure factor has an isotropic ring pattern, as shown in Fig. 5 (A), indicating the liquid phase. The phase behavior of the PS particle monolayer would switch into another phase as the reduced density is increased. In Fig. 5(D)-(F), the structure factors have a hexagonal pattern with blurry spots to distinguish this phase as the so-called "hexatic phase". On the other hand, at high reduced densities, say $\rho^{*}>0.043$, the two-dimensional structure factor has a clearly defined hexagonal pattern of spots, as shown in Fig. 4(G) and $(\mathrm{H})$, indicating the solid phase. Note that the solid phase occurs at quite low densities, in contrast to the quasi-two-dimensional system of Rice and coworkers [6,7] in which the solid phase occurs at relatively high reduced densities: 0.874 for PMMA particles and 0.704 for silica particles. These authors have found that the phase transition behavior is strongly related to the interaction between particles. The interaction can be adjusted by grafting the particles with diverse functional groups. In quasi- two-dimensional system, colloidal particles were suspended in aqueous phase. The position of the first peak in the radial distribution function stands for the nearest neighbor distance, which is always smaller than the distance of two times diameter in each phase. That indicates the interaction between two particles is quite short range [6,7]. In our system, PS particles were adsorbed at decane/water interface, the interfacial tension between PS particle and decane, besides the interfacial tension between particle and water, also plays a role in the interaction between particles. According to our experimental results of the radial distribution function, the nearest neighbor distance in the solid phase is about 5 times diameter and 15 times diameter in the liquid phase which implies the interaction is in a much longer range than that in quasi-two-dimensional system. It is interesting that the system exhibits a sequence of phase transitions: liquid phase $\rightarrow$ hexatic phase $\rightarrow$ solid phase, as the density is increased, as shown in Figs. 4 and 5.

There are several advantages of using Langmuir trough as the platform to study two-dimensional particle monolayer. First of all, we can easily get different densities by simply compressing the barriers. Second, boundary effects could be avoided, in contrast to the wall drag effect in the system of Rice and coworkers [6-8]. The most important issue is that the Langmuir trough is much easier to prepare. One of the advantages of applying the Langmuir trough is that the Langmuir trough could provide the information of surface pressure as a function of the reduced density. That is, the isotherm of the particle monolayer should exhibit a distinct feature as a phase transition occurs. Unfortunately, in our system, the reduced density of the system is too dilute to induce the change of surface pressure substantially. The surface pressure remains almost constant and no significant change of surface pressure is observed during the compression, consistent with the results of Aveyard et al. [10]. Note that in the whole compression process of the experiments of Aveyard et al. [10], only the hexagonal structure was observed. That is, all these experiments [10] fall into the solid phase regime. In all these previous studies using the Langmuir trough [10-13], no phase transition has been mentioned and discussed before. It is the first study, to the best of our knowledge, to observe a sequence of phase transitions occurring at quite low densities by simply using the Langmuir trough. In addition, these transitions cannot be distinguished by surface pressure.

\section{Conclusion}

A simple and convenient method to observe phase transitions in two-dimensional colloidal particles by applying the Langmuir trough is presented in this work. A sequential phase transition, liquid phase $\rightarrow$ hexatic phase $\rightarrow$ solid phase, is observed in the system of the polystyrene particle ( $2 \mu \mathrm{m}$ in diameter) monolayer as a function of particle density at $20^{\circ} \mathrm{C}$.

\section{Acknowledgement}

This work was supported by the National Science Council of Taiwan, ROC. 


\section{References}

[1] C.A. Murray, D.H.V. Winkle, Phys. Rev. Lett. 58 (1987) 1200.

[2] E. Sheppard, N. Tcheurekdjian, J. Colloid Interface Sci. 28 (1968) 481.

[3] P. Hanarp, D.S. Sutherland, J. Gold, B. Kasemo, Colloids Surf. 214 (2003) 23-36.

[4] Z. Horvolgyi, M. Mate, M. Zrinyl, Colloids Surf. A 84 (1994) 207.

[5] S. Slomkowski, D. Kowalczyk, M. Trznadel, Trends Polym. Sci. 3 (1995) 297-304.

[6] B. Cui, B. Lin, S.A. Rice, J. Chem. Phys. 114 (2001) 9142-9155.
[7] A.H. Marcus, S.A. Rice, Phys. Rev. E 55 (1997) 637.

[8] B. Lin, J. Yu, S.A. Rice, Phys. Rev. E 62 (2000) 3909.

[9] J.C. Crocker, D.G. Grier, J. Colloid Interface Sci. 179 (1996) 298.

[10] R. Aveyard, J.H. Clint, D. Nees, V.N. Paunov, Langmuir 16 (2000) 1969-1979.

[11] R. Aveyard, J.H. Clint, D. Nees, N. Quirke, Langmuir 16 (2000) 8820-8828.

[12] B.P. Binks, S.O. Lumsdon, Langmuir 16 (2000) 8622-8631.

[13] B.P. Binks, P.D.I. Fletcher, Langmuir 17 (2001) 4708-4710. 